\title{
Simplest Method for Calculating the Lowest Achievable Uncertainty of Model at Measurements of Fundamental Physical Constants
}

\author{
Boris Menin \\ Refrigeration Consultancy Ltd., Beer-Sheba, Israel \\ Email: meninbm@gmail.com
}

How to cite this paper: Menin, B. (2017) Simplest Method for Calculating the Lowest Achievable Uncertainty of Model at Measurements of Fundamental Physical Constants. Journal of Applied Mathematics and Physics, 5, 2162-2171.

https://doi.org/10.4236/jamp.2017.511176

Received: October 16, 2017

Accepted: November 7, 2017

Published: November 10, 2017

Copyright $\odot 2017$ by author and Scientific Research Publishing Inc. This work is licensed under the Creative Commons Attribution International License (CC BY 4.0).

http://creativecommons.org/licenses/by/4.0/

\begin{abstract}
The CODATA procedure for calculating the recommended relative uncertainty of the measured fundamental physical constants is complex and is based on the use of powerful computers and modern mathematical statistical methods. In addition, the expert's opinion caused by accumulated knowledge, life experience and intuition of researchers is applied at each stage of the calculations. In this article, the author continues to advocate a theoretically grounded information method as the most effective tool for testing and achieving the minimum possible relative uncertainty for any measurements of experimental physics and engineering. The introduced fundamental limit characterizing discrepancy between a model and the observed object cannot be overcome by any improvement of instruments, methods of measurement and the model's computerization. Examples are given.
\end{abstract}

\section{Keywords}

Fundamental Physical Constants, Information Theory, Mathematical Modeling, Similarity Theory, Uncertainty

\section{Introduction}

In the past century, an increased attention to the accuracy of fundamental physical constants has led to the development of new physical theories and many technological improvements [1]. We are, however, possibly gradually reaching the limits of accuracy, in spite of the fact of usage of powerful computers and unique newest mathematical methods. Is there a reasonable limit to our exact 
understanding surrounding the world? This limit is unquestionably manifested in the restructuring of the International System of Units (SI) and in the identification of the difficulties that now appear in electrical measurements [2].

Why is it important to know the limits of our ability to measure fundamental physical constants? First, a precise definition of the fundamental constants allows us to verify the consistency and correctness of the basic physical theories. Second, the quantitative predictions of the basic physical theories depend on the numerical values of the constants involved in these theories: each new sign can lead to the discovery of a previously unknown inconsistency or, conversely, can eliminate the existing inconsistency in our description of the physical world. However, by formulating the model of the experiment, scientists, on the one hand, somehow break off the connections with possible, potentially influencing variables, which are hidden from our eyes at the moment. On the other hand, it is very difficult to measure all the variables that are taken into account in the measurement model with high accuracy and not 'miss' the significant effect. In addition to these problems, it often turns out that a new series of measurements using more advanced methods shows that the former value was erroneous and that its new value differs from the old 'conventional' by an amount many times greater than the uncertainty attributed to the previous value.

These 'flowers' do not end the field of activity of scientists to clarify the true meaning of fundamental physical constants. A difficult task in negotiating the values of constants is the estimation of uncertainties. In most experiments, physicists try to collect as much data as possible in order to reduce the random measurement uncertainty to a negligible value. In this case, the final uncertainty attributed to the result of the measurement is determined only based on an assessment of systematic uncertainties. These uncertainties are associated with effects, for which little is known. Therefore, the corresponding estimates are somewhat subjective and are usually obtained essentially intuitively [1]. The problem is aggravated by the fact that different experimenters approach the evaluation of systematic uncertainties from completely different positions. Some of them cautiously attribute their data to an overestimated uncertainty in the hope that subsequent measurements will not reject their results as incorrect. Others, on the contrary, underestimate the sources of systematic uncertainties in their experiments, apparently proceeding from an unconscious (and perhaps even intentional) desire to conduct 'the best experiment'. Such factors, so far from scientific objectivity, are inevitable, since in the end, people who have different life experiences and are endowed with greater or lesser abilities make science. That is why the measurement uncertainties presented by different researchers are not at all easy to compare with each other.

Undoubtedly, the coordinated set of physical constants of 2015 [3] is closer to the truth than the previous ones. However, being realists, we cannot reject the possibility that a new, theoretically grounded approach to the calculation of the recommended value of the relative uncertainty is required or can exist, by reali- 
zation of which measuring of the true-target values of fundamental physical constants is possible.

That is why, from the point of view of the author, the recently developed information approach may to some extent facilitate the process of calculating the amount of relative uncertainty to be measured by physicists. It is based on the proposition that a multi-physics model contains a certain quantity of information about the object under study, depending on the quantitative and qualitative set of physical variables to be taken into account. By this way the optimal number of selected parameters can be calculated. It allows reaching the lowest discrepancy between a model and the observed object. This approach defines a limit of accuracy that cannot be overcome by any improvement of instruments, methods of measurement and the model's computerization. It has physical meaning and its value is much higher than the Heisenberg uncertainty relation provides. It is important to mention that applying information theory allows giving a theoretical explanation and grounding of experimental results, which determine precision of fundamental constants.

The information approach plays a decisive role in a new view of the achievement of the least uncertainty in the model of a physical phenomenon. Unfortunately, it is still little known to most scientists and engineers. In this paper, we continue to apply it for measurements of fundamental physical constants.

\section{Suggested Applied Tools}

In [4], it was shown that a certain uncertainty exists before starting an experiment or computer simulations. It is caused due only to the known number of recorded variables. The value of this uncertainty can be calculated by the following formula

$$
\Delta_{\mathrm{pmm}}=S \cdot\left[\left(\mathrm{z}^{\prime}-\beta^{\prime}\right) / \mu_{\mathrm{SI}}-\left(\mathrm{z}^{\prime \prime}-\beta^{\prime \prime}\right) /\left(\mathrm{z}^{\prime}-\beta^{\prime}\right)\right],
$$

where $u$ is the dimensionless researched variable; $\Delta u$ is the dimensionless uncertainty of the physical-mathematical model describing the experiment with the apriority chosen number of variables; $S$ is the predetermined dimensionless interval of $u$ variations; $z^{\prime \prime}$ is the given number of selected physical dimensional variables; $\beta^{\prime \prime}$ is the number of primary physical variables recorded in a model; $\varepsilon=\Delta_{\mathrm{pmm}} / S$ is the comparative uncertainty. Equation (1), surprisingly, is very simple. Absolute and relative uncertainties are familiar to physicists. As for the comparative uncertainty, it is rarely mentioned. Nevertheless, the importance of comparative uncertainty is of great importance for the application of information theory in physics and engineering; $z^{\prime}$ is the number of physical dimensional variables in the selected class of phenomena ( $C o P$-see below), $\beta^{\prime}$ is the number of primary physical dimensional variables in the selected $C o P ; \mu_{\mathrm{SI}}$ is the total number of possible dimensionless criteria with $\xi=7$ main dimensional variables for SI-see below.

The relation (1), which follows from the general provisions of information theory, is accurate and does not depend on the conditions of experience, the 
concrete implementation of the test stand, the expert opinion of scientists and the selected statistical mathematical methods.

An overall uncertainty of the model including inaccurate input data, physical assumptions, the approximate solution of the integral-differential equations, etc., will be larger than $\Delta_{\mathrm{pmm}}$. Thus, $\Delta_{\mathrm{pmm}}$ is the first-born and least component of a possible mismatch of a real object and its modeling results.

Equation (1) has physical meaning. It testifies that in nature there is a fundamental limit to the accuracy of measuring any process, which cannot be surpassed by any improvement of instruments, methods of measurement and the model's computerization. The value of this limit is much higher and stronger than the Heisenberg uncertainty relation provides. In addition, this fundamental limit places severe restrictions on the micro-physics.

SPV and SI are a fantasy generated by collective imagination. However, without SPV, the simulation of the phenomenon is impossible. You can interpret SPV as the basis of all the available knowledge that people have about the surrounding nature at the moment.

SI includes the primary and secondary variables used for descriptions of different classes of phenomena $(C o P)$. For example, in mechanics of SI there is used a basis $\{L$-length, $M$-mass, $T$-time $\}$, i.e. $C o P_{\mathrm{SI}} \equiv L M T$.

It is known [5] [6] that the dimension of any secondary variable can be expressed as a unique function of the product of primary variables $L, M, T, I, \Theta, J$, and $F$ with certain exponents $I, m, t, i, \theta, j, f$, which can take only integer values and vary in specific ranges

$$
\begin{gathered}
q \ni L^{l} \cdot M^{m} \cdot T^{t} \cdot I^{i} \cdot \Theta^{\theta} \cdot J^{j} \cdot F^{f}, \\
-3 \leq l \leq+3,-1 \leq m \leq+1,-4 \leq t \leq+4,-2 \leq i \leq+2, \\
-4 \leq \theta \leq+4,-1 \leq j \leq+1,-1 \leq f \leq+1, \\
e_{l}=7 ; e_{m}=3 ; e_{t}=9 ; e_{i}=5 ; e_{\theta}=9 ; e_{j}=3 ; e_{f}=3
\end{gathered}
$$

where $e_{1}, \cdots, e_{f}$ are the numbers of variants of the dimension for each variable. For example, $I^{3}$ is used in the density formula; $\theta^{4}$ is used in the Stefan-Boltzmann law.

We can calculate the total number of possible dimensionless criteria $\mu_{\mathrm{SI}}$ with $\xi=7$ main dimensional variables for SI [4]

$$
\mu_{\mathrm{SI}}=\Psi-\xi=38272-7=38265
$$

where $\Psi=38,272$ is the total number of dimensional options of physical variables in SI; $\mu_{\mathrm{SI}}$ corresponds to the maximum amount of information contained in the SPV; each variable allows the researcher to obtain a certain amount of information about the studied object; the main definitions and estimates of the amount of information used in the experiment were clearly formulated by $\mathrm{L}$. Brillouin [7] and generalized by M. Burgin [8].

Equating the derivative of $\Delta_{\mathrm{pmm}} / S$ from Equation (1) with respect to $z^{\prime}-\beta^{\prime}$ to zero, we obtain the condition for achieving the minimum comparative uncertainty for a particular COP: 


$$
\left(z^{\prime}-\beta^{\prime}\right)^{2} /(\Psi-\xi)=\left(z^{\prime \prime}-\beta^{\prime \prime}\right) .
$$

For the analysis of experimental data, we need to know the recommended number of selectable variables, with which we can achieve a minimum comparative uncertainty for a specific $C o P$ :

1) For $C o P_{\mathrm{SI}} \equiv L M T F$, taking into account the aforementioned explanations and (6), the lowest comparative uncertainty $\mathcal{E}_{L M T F}$ can be reached at the following conditions:

$$
\begin{gathered}
\left(z^{\prime}-\beta^{\prime}\right)=\left(e_{l} \times e_{m} \times e_{t} \times e_{f}-1\right) / 2-4=(7 \times 3 \times 9 \times 3-1) / 2-4=279 \\
\left(z^{\prime \prime}-\beta^{\prime \prime}\right)=\left(z^{\prime}-\beta^{\prime}\right)^{2} / \mu_{\mathrm{SI}}=279^{2} / 38265 \approx 2
\end{gathered}
$$

where ' -1 ' corresponds to the case when all the primary variable exponents are zero in Formula (2); dividing by 2 indicates that there are direct and inverse variables, e.g., $L^{1}$ is length, $L^{-1}$ is run length. Because the object can be judged knowing only one of its symmetrical parts, while others structurally duplicating this part may be regarded as information empty [4]. Therefore, the number of options of dimensions may be reduced by $\omega=2$ times; 4 corresponds to the four primary variables $L, M, T, F$.

$$
\begin{aligned}
\left(\varepsilon_{\text {min }}\right)_{\text {LMTF }} & =\Delta_{\mathrm{pmm}} / S=\left[\left(z^{\prime}-\beta^{\prime}\right) / \mu_{\mathrm{SI}}-\left(z^{\prime \prime}-\beta^{\prime \prime}\right) /\left(z^{\prime}-\beta^{\prime}\right)\right] \\
& =279 / 38265+2 / 279=0.0073+0.0073=0.0146
\end{aligned}
$$

2) For $C o P_{\mathrm{SI}} \equiv L M T \theta I$, taking into account (6), the lowest comparative uncertainty $\varepsilon_{L M T F I}$ can be reached at the following conditions:

$$
\begin{aligned}
& \left(z^{\prime}-\beta^{\prime}\right)_{L \text { LM } \theta I}=\left(e_{1} \times e_{m} \times e_{t} \times e_{\theta} \times e_{i}-1\right) / 2-5=(7 \times 3 \times 9 \times 9 \times 5-1) / 2-5=4247 \\
& \left(z^{\prime \prime}-\beta^{\prime \prime}\right)_{L M T \theta I}=\left(z^{\prime}-\beta^{\prime}\right)^{2} / \mu_{\mathrm{SI}}=4247^{2} / 38265 \approx 471
\end{aligned}
$$

where ' -1 ' corresponds to the case when all the primary variable exponents are zero in Formula (2); dividing by 2 indicates that there are direct and inverse variables, e.g., $L^{1}$-length, $L^{-1}$-run length, and 5 corresponds to the five primary variables $L, M, T, \Theta, I$.

Then, one can calculate the minimum achievable comparative uncertainty $\mathcal{E}_{L M T \Theta I}$

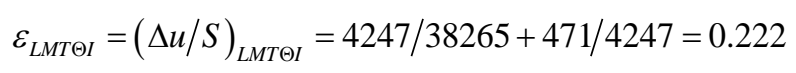

Let's speculate about applying the information approach for the measurement of several fundamental physical constants.

\section{Applications}

\subsection{Proton Mass $m_{p}$}

We analyzed several research publications and CODATA (Committee on Data for Science and Technology) recommendations over the past 19 years (Table 1, [9]-[15]) from the position of the reached relative uncertainty values. All studies belong to the $C o P_{\mathrm{SI}} \equiv L M T \theta I$. In none of the current experiments of the calculation of the $m_{p}$ value has the prospective interval been declared, in which its true 
Table 1. Proton mass and achieved relative uncertainty.

\begin{tabular}{crrcc}
\hline No & Year & Proton mass $\times 10^{27}, \mathrm{~kg}$ & Achieved relative uncertainty & References \\
\hline 1 & 1999 & 1.6726217161 & $4.6 \times 10^{-7}$ & {$[9]$} \\
2 & 2005 & 1.6726217129 & $1.7 \times 10^{-7}$ & {$[10]$} \\
3 & 2008 & 1.6726216378 & $5 \times 10^{-8}$ & {$[11]$} \\
4 & 2008 & 1.6726217162 & $1.8 \times 10^{-10}$ & {$[12]$} \\
5 & 2012 & 1.6726217777 & $4.4 \times 10^{-8}$ & {$[13]$} \\
6 & 2014 & 1.6726218982 & $1.2 \times 10^{-8}$ & {$[14]$} \\
7 & 2017 & 1.6726217154 & $3.2 \times 10^{-11}$ & {$[15]$} \\
\hline
\end{tabular}

value can be placed. In other words, the exact trace of the placement of $m_{p}$ is lost somewhere. Therefore, in order to apply our stated approach, as a possible measurement interval of $m_{p}$, we choose the difference of its value reached by the experimental results of two projects: $m_{p \min }=1.67262163783 \times 10^{-27} \mathrm{~kg}$ [11] and $m_{p \max }=1.67262189821 \times 10^{-27} \mathrm{~kg}$ [14]. Then, the possible observed range $S_{p}$ of $m_{p}$ variations equals

$$
\begin{aligned}
S_{p}= & m_{p \max }-m_{p \min }=1.67262189821 \times 10^{-27} \\
& -1.67262163783 \times 10^{-27}=2.6 \times 10^{-34}(\mathrm{~kg})
\end{aligned}
$$

It is seen from the data given in Table 1 that there was not dramatic improvement of the measurement accuracy of $m_{p}$ during the last 18 years by view of the relative uncertainty, except [15]. It differs sharply from other calculated values of relative uncertainty. The question of reliability is key, since the refinement of the values of fundamental constants by innovative methods is extremely vulnerable [2]. Although specialists are highly qualified and use the latest technologies, the lack of accumulated experience in pioneering research affects and we need to wait new experiments.

It is obvious that the spread in the magnitude of the measured $m_{p}$ is significant. In addition, the truthful and precise value of $m_{p}$ is not known at the moment. Therefore, scientists of CODATA calculate and declare each 2 years the recommended value of the relative uncertainty, by which, in the future, it will be possible to achieve the true-target value of $m_{p}$.

We can argue about the order of the desired value of the relative uncertainty of $C o P_{\mathrm{SI}} \equiv L M T \Theta I$ that is usually used for measurements of the proton mass. For this purpose, we take into account $\left(\varepsilon_{\min }\right)_{L M T \theta I}=0.222, S_{p}=2.6 \times 10^{-34} \mathrm{~kg}$. Then, the lowest possible absolute uncertainty for $C o P_{\mathrm{SI}} \equiv L M T \Theta I$ equals

$$
\left(\Delta_{\min }\right)_{L M T \theta I}=\left(\varepsilon_{\min }\right)_{L M T \theta I} \times S_{p}=0.222 \times 2.6 \times 10^{-34}=0.5772 \times 10^{-34}(\mathrm{~kg})
$$

In this case, the lowest possible relative uncertainty $\left(r_{\min }\right)_{L M T \theta I}$ for $\operatorname{Co} P_{\mathrm{SI}} \equiv$ $L M T \Theta I$ is as follows:

$$
\begin{aligned}
\left(r_{\min }\right)_{\text {LMT } \theta I} & =\left(\Delta_{\min }\right)_{L_{\text {LMT } \theta I}} /\left(\left(m_{p \max }+m_{p \min }\right) / 2\right) \\
& =0.5772 \times 10^{-34} /\left(1.672621768 \times 10^{-27}\right)=3.4 \times 10^{-8}
\end{aligned}
$$


This value is in excellent agreement with the recommendations mentioned in [13] $\left(4.4 \times 10^{-8}\right)$, and can be used for the new definition of the Kelvin and a significant revision of the International System of Units.

\subsection{Avogadro Number Na}

We performed an analogous procedure for analyzing the results of measurements of the Avogadro number over the past 15 years ([11] [13] [14] [16]-[20]). The data are summarized in Table 2.

All studies belong to the $C o P_{\mathrm{SI}} \equiv L M T F$. In order to verify the desired value of the relative uncertainty $\left(r_{\min }\right)_{L M T F}$ of $C o P_{\mathrm{SI}} \equiv L M T F$ and taking into account (1) (4) (5) (7) (8), we get the following:

$$
\begin{aligned}
\left(z^{\prime}-\beta^{\prime}\right)_{L M T F}=\left(e_{l} \times e_{m} \times e_{t} \times e_{f}-1\right) / 2-4=(7 \times 3 \times 9 \times 3-1) / 2-4=279 \\
\left(z^{\prime \prime}-\beta^{\prime \prime}\right)_{L M T F}=\left(z^{\prime}-\beta^{\prime}\right)^{2} / \mu_{\mathrm{SI}}=279^{2} / 38265 \approx 2 \\
\left(\varepsilon_{\min }\right)_{L M T F}=\Delta_{\mathrm{pmm}} / S=\left[\left(z^{\prime}-\beta^{\prime}\right) / \mu_{\mathrm{SI}}-\left(z^{\prime \prime}-\beta^{\prime \prime}\right) /\left(z^{\prime}-\beta^{\prime}\right)\right] \\
=279 / 38265+2 / 279=0.0073+0.0073=0.0146 \\
N_{a \max }=6.022141793 \times 10^{23}\left(\mathrm{~mol}^{-1}\right) \quad[14], \\
N_{a \min }=6.022133900 \times 10^{23}\left(\mathrm{~mol}^{-1}\right)[11], \\
S_{N a}=\left(N_{a \max }-N_{a \min }\right)=7.9 \times 10^{17}\left(\mathrm{~mol}^{-1}\right) \\
\left(\Delta_{\min }\right)_{L M T F}=\left(\varepsilon_{\min }\right)_{L M T F} \times\left(N_{a \max }-N_{a \min }\right) \\
=0.0146 \times 7.9 \times 10^{17}=0.1153 \times 10^{17}\left(\mathrm{~mol}^{-1}\right) \\
\left(r_{\min }\right)_{L M T F}=\left(\Delta_{\min }\right)_{L M T F} /\left(\left(N_{a \max }+N_{a \min }\right) / 2\right) \\
=0.1153 \times 10^{17} /\left(6.0221378465 \times 10^{23}\right)=1.9 \times 10^{-8}
\end{aligned}
$$

The value $1.9 \times 10^{-8}$ is in excellent agreement with the recommendations mentioned in [20] $\left(2 \times 10^{-8}\right)$, and can be used for a significant revision of the International System of Units.

It is necessary to note the fundamental difference between the described method and the CODATA method for determining the recommended value of the

Table 2. Avogadro number and achieved relative uncertainty.

\begin{tabular}{ccccc}
\hline $\mathrm{N}$ & Year & Value of $N_{a} \times 10^{-23}, \mathrm{~mol}^{-1}$ & Achieved relative uncertainty $\times 10^{8}$ & References \\
\hline 1 & 2001 & 6.022133900 & 46 & {$[16]$} \\
2 & 2003 & 6.022135300 & 34 & {$[17]$} \\
3 & 2008 & 6.0221417930 & 5 & {$[11]$} \\
4 & 2011 & $6.02214082(18)$ & 3 & {$[18]$} \\
5 & 2011 & $6.02214078(18)$ & 3 & {$[19]$} \\
6 & 2012 & $6.02214129(27)$ & 4.4 & {$[13]$} \\
7 & 2014 & $6.022140857(74)$ & 1.2 & {$[14]$} \\
8 & 2015 & $6.02214076(12)$ & 2 & {$[20]$} \\
\hline
\end{tabular}


relative uncertainty of a fundamental physical constant. Within the framework of the CODATA concept, a detailed discussion of the input data and the justification and construction of tables of values sufficient for the direct use of relative uncertainty are conducted using modern advanced statistical methods and powerful computers. This, in turn, allows you to check the self-consistency of input data and output sets of values. However, at each stage of data processing, an expert conclusion based on intuition, accumulated knowledge and the life experience of scientists is also used. Within the framework of the presented approach, a theoretical and informational justification is carried out to calculate the relative uncertainty. A detailed description of the data and the processing procedures used do not require considerable time. This is a reason for the wide implementation of the $\mu_{\mathrm{SI}}$-hypothesis, the concept of a system of primary variables for analyzing existing experimental data on the measurement of fundamental physical constants.

\section{Conclusions}

The information approach for calculating the uncertainty of the model of a physical phenomenon or technological process is very promising in those areas of science and technology where it is required to predict the result of an experiment or to calculate a given basic parameter with very high accuracy, for example, reliability of an atomic power station, seismic stability of buildings, strength of a submarine's hull, thermal resistance of spacecraft's casing, measurement of fundamental physical constants and so on.

The 'new angle' is to apply an information approach to the problems associated with the origin of the choice of the most applicable value of the recommended relative uncertainty. We think that this is perhaps the only tool that does this strictly theoretically.

This is in a sense a little bit more basic as Heisenberg tenet declares. The fundamental limit places severe restrictions on the micro-physics.

We hope that the implementation of the information approach will be recreated in real experiments, possibly using appropriate test benches and various groups of variables. But even if finely tuned fixed points can be provided in the laboratory, we view the presented method as 'necessary but not sufficient', because, at this moment, it cannot accurately indicate the true set of specific variables to achieve a minimum comparative uncertainty.

In our view, the ability of our approach to respond to information quantity embedded in a model is key that can get up and walk away from the subjective environment consisting from accumulated knowledge, life experience and intuition of scientists.

Even if the information approach is on the right track about experimental physics and technology, we want and will develop more detailed information, such as a theory about which primitive 'proto-bricks' are at the core of the system of primary variables, and how this system can be extended and modified, close to the surrounding natural perfection or chaos. 


\section{References}

[1] Taylor, B.N., Langenberg, D.N. and Parker, W.H. (1970) The Fundamental Physical Constants. Scientific American, 223, 62-78. https://goo.gl/jZ55TD

[2] Karshenboim, S.G. (2013) Progress in the Accuracy of the Fundamental Physical Constants: 2010 CODATA Recommended Values. UFN, 183, 935-962. http://www.mathnet.ru/links/fb8357c7049e641ede305b7d35c7578f/ufn4438.pdf

[3] Mohr, P.J., Taylor, B.N. and Newell D.B. (2016) CODATA Recommended Values of the Fundamental Physical Constants: 2014. Reviews of Modern Physics, 88, 1-73. http://ws680.nist.gov/publication/get_pdf.cfm?pub_id=920687

[4] Menin, B. (2017) Universal Metric for the Assessing the Magnitude of the Uncertainty in the Measurement of Fundamental Physical Constants. Journal of Applied Mathematics and Physics, 5, 365-385. http://file.scirp.org/pdf/JAMP_2017021711410991.pdf

[5] NIST Special Publication 330 (SP330), the International System of Units (SI), 2008, $1-97$. http://nvlpubs.nist.gov/nistpubs/Legacy/SP/nistspecialpublication330e2008.pdf

[6] Sonin, A.A. (2001) The Physical Basis of Dimensional Analysis. 2nd Edition, Department of Mechanical Engineering, MIT, Cambridge, 1-57. http://web.mit.edu/2.25/www/pdf/DA_unified.pdf

[7] Brillouin, L. (1964) Scientific Uncertainty and Information. Academic Press, New York, 1-155. https://goo.gl/JbPFvF

[8] Burgin, M. (2009) Theory of Information-Fundamentality, Diversity and Unification. World Scientific Publishing Co., USA 2009, ch. 3, 255-300.

[9] Van Dyck, R.S., Farnham, Jr. D.L., Zafonte, S.L. and Schwinberg, P.B. (1999) High precision Penning Trap Mass Spectroscopy and a New Measurement of the Proton's “Atomic Mass". In: Dubin D.E. and Schneider, D., Eds., Trapped Charged Particles and Fundamental Physics, AIP Conference Proceedings 457 AIP, Woodbury, NY, 101, 2-11. https://goo.gl/VFxkVu

[10] CODATA Recommended Values of the Fundamental Physical Constants: 2002, 1-107. https://goo.gl/ZJsZQL

[11] Mohr, P.J., Taylor, B.N. and Newell, D.B. (2008) CODATA Recommended Values of the Fundamental Physical Constants: 2006. Reviews of Modern Physics, 80, 1-98. https://goo.gl/h5Sc1P

[12] Solders, A., Bergström, I., Nagy, Sz., Suhonen, M. and Schuch, R. (2008) Determination of the Proton Mass from a Measurement of the Cyclotron Frequencies of $\mathrm{D}^{+}$ and $\mathrm{H}_{2}{ }^{+}$in a Penning Trap. Physical Review Letters A, 78, Article ID: 012514. https://goo.gl/5CXswV

[13] Mohr, P.J., Taylor, B.N. and Newell, D.B. (2012) CODATA Recommended Values of the Fundamental Physical Constants: 2010. Journal of Physical and Chemical Reference Data, 41, Article ID: 043109. https://goo.gl/tqwqoy

[14] CODATA Recommended Values of the Fundamental Physical Constants: 2014. https://codata.org/blog/?p=451

[15] Heiße, F., Köhler-Langes, F., Rau, S., Hou, J., Junck, S., Kracke, A., Mooser, A., Quint, W., Ulmer, S., Werth, G., Blaum, K. and Sturm, S. (2017) High-Precision Measurement of the Proton's Atomic Mass. Physical Review Letters, 119, Article ID: 033001. https://goo.gl/iRozRW

[16] De Bievre, P., Valkiers, S., Kessel, R., Taylor, P.D.P., Becker, P., Bettin, H., Peuto, A., 
Pettorruso, S., Fujii, K., Waseda, A., Tanaka, M., Deslattes, R.D., Peiser, H.S. and Kenny, M.J. (2001) A Reassessment of the Molar Volume of Silicon and of the Avogadro Constant. IEEE Transactions on Instrumentation and Measurement, 50, 593-597. https://goo.gl/hCJkce

[17] Becker, P., Bettin, H., Danzebrink, H.-U., Gläser, M., Kuetgens, U., Nicolaus, A., Schiel, D., De Bièvre, P., Valkiers, S. and Taylor, P. (2003) Determination of the Avogadro Constant via the Silicon Route. Metrologia, 40, 271-287. https://goo.gl/vp88c5

[18] (2011) International Avogadro Project. Physical Review Letters, 106, Article ID: 030801. https://goo.gl/33E6Z3

[19] Andreas, B., Azuma, Y., Bartl, G., Becker, P., Bettin, H., Borys, M., Busch, I., Gray, M., Fuchs, P., Fujii, K., Fujimoto, H., Kessler, E., Krumrey, M., Kuetgens, U., Kuramoto, N., Mana, G., Manson, P., Massa, E. and Mizushima, S. (2011) Determination of the Avogadro Constant by Counting the Atoms in a ${ }^{28} \mathrm{Si}$ Crystal. Physical Review Letters, 106, Article ID: 030801. https://goo.gl/4g7nkr

[20] Azuma, Y., et al. (2015) Improved Measurement Results for the Avogadro Constant using a ${ }^{28}$ Si-Enriched Crystal. Metrologia, 52, 360-375. https://goo.gl/PURKaG 